\title{
Spectroscopic applications and frequency locking of THz photomixing with distributed-Bragg-reflector diode lasers in low-temperature-grown GaAs
}

\author{
Pin Chen a) and Geoffrey A. Blake \\ Division of Chemistry and Chemical Engineering, California Institute of Technology, M/S 127-72, \\ Pasadena, California 91125
}

M. C. Gaidis

M/S 168-314, Jet Propulsion Laboratory, Pasadena, California 91109

\author{
E. R. Brown and K. A. McIntosh \\ Lincoln Laboratory, Massachusetts Institute of Technology, Lexington, Massachusetts 02173-9108
}

S. Y. Chou, M. I. Nathan, and F. Williamson

Department of Electrical Engineering, University of Minnesota, Minneapolis, Minnesota 55455

(Received 7 May 1997; accepted for publication 10 July 1997)

\begin{abstract}
A compact, narrow-linewidth, tunable source of $\mathrm{THz}$ radiation has been developed for spectroscopy and other high-resolution applications. Distributed-Bragg-reflector (DBR) diode lasers at $850 \mathrm{~nm}$ are used to pump a low-temperature-grown GaAs photomixer. Resonant optical feedback is employed to stabilize the center frequencies and narrow the linewidths of the DBR lasers. The heterodyne linewidth full-width at half-maximum of two optically locked DBR lasers is $50 \mathrm{kHz}$ on the $20 \mathrm{~ms}$ time scale and $2 \mathrm{MHz}$ over $10 \mathrm{~s}$; free-running DBR lasers have linewidths of 40 and 90 $\mathrm{MHz}$ on such time scales. This instrument has been used to obtain rotational spectra of acetonitrile $\left(\mathrm{CH}_{3} \mathrm{CN}\right)$ at $313 \mathrm{GHz}$. Detection limits of $1 \times 10^{-4} \mathrm{~Hz}^{1 / 2}$ (noise/total power) have been achieved, with the noise floor dominated by the detector's noise equivalent power. () 1997 American
\end{abstract} Institute of Physics. [S0003-6951(97)00538-X]

Recent advances in the development of low-temperaturegrown (LTG) GaAs as a THz optical heterodyne photomixer, when combined with near-infrared semiconductor lasers, promise a coherent radiation source that is continuously tunable, compact, robust, and inexpensive. ${ }^{1-4}$ Such a frequency synthesis technique would benefit many $\mathrm{THz}$ applications including frequency metrology of near-infrared spectral lines, air/space-borne measurements for atmospheric and astrophysical studies, and high resolution molecular spectroscopy. A handful of groups ${ }^{1-4}$ have developed LTG GaAs based $\mathrm{THz}$ sources. However, these sources suffer the disadvantages of having a cumbersome size, a limited tuning range, frequency instabilities, and/or expensive pump lasers. Through the use of resonant optical feedback ${ }^{5-7}$ to lock the frequency of two distributed-Bragg-reflector (DBR) diode pump lasers, we have achieved a tunable source that is inexpensive, compact, narrow linewidth, and frequency stable.

Figure 1 illustrates the experimental setup. The LTG GaAs photomixer is pumped by two optically locked DBR diode lasers. We use the same geometry developed by Hollberg and co-workers. ${ }^{5}$ A small fraction of each DBR laser's output is sent to a folding mirror ( $\phi$ mirror) and then to a confocal Fabry-Perot cavity. The DBR lasers "see" optical feedback only when they are on resonance with the FabryPerot cavities. Thus, narrowing of the linewidth via optical feedback is achieved in a controlled manner. Each cavity consists of two spherical mirrors glued onto quartz and piezoelectric translator (PZT) tubes. The PZT section allows one to tune the cavity-free spectral range [(FSR),

${ }^{a}$ Electronic mail: pin@gps.caltech.edu

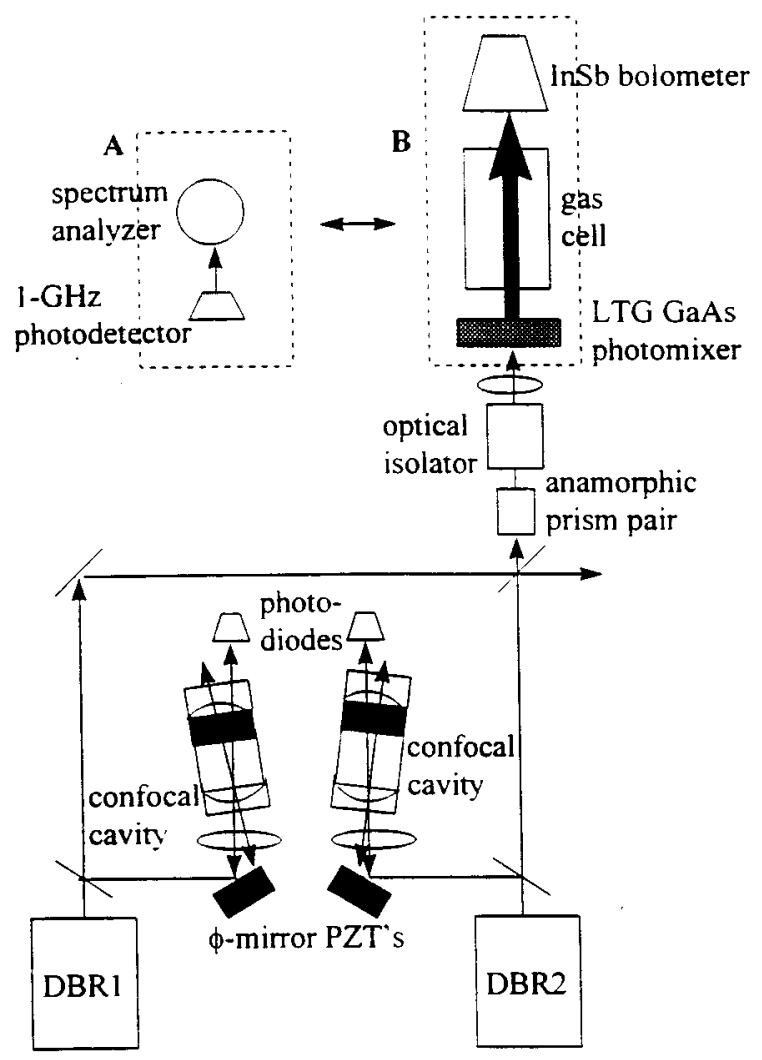

FIG. 1. The experimental setup. Each of the two DBR lasers is optically locked to an independent confocal cavity. PZT translators tune the cavity lengths and the feedback path lengths ( $\phi$-mirror positions). The primary beams from the DBR lasers are combined by a beamsplitter. The combined beam goes through either $\mathbf{A}$ for heterodyne linewidth measurements, or $\mathbf{B}$ for submillimeter spectroscopy. 


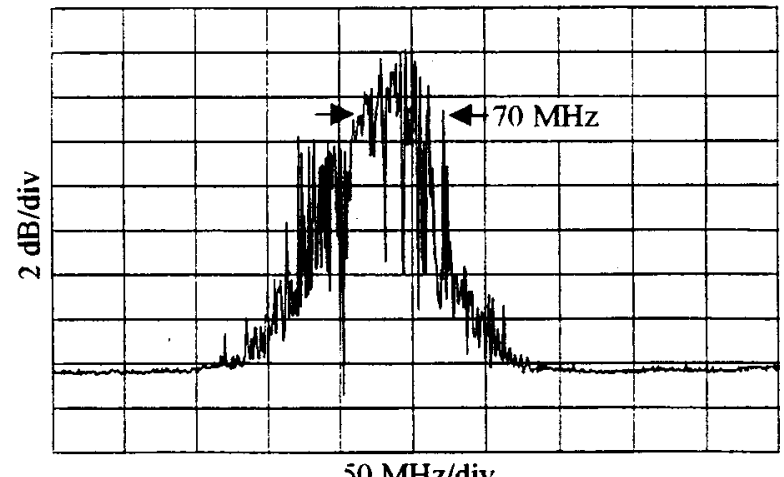

$50 \mathrm{MHz} / \mathrm{div}$

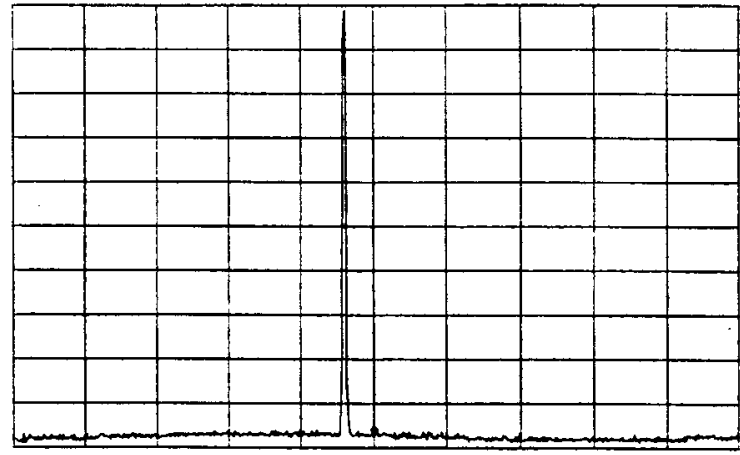

FIG. 2. The long-term frequency stability. Upper plot is the heterodyne beatnote of two free-running DBR lasers. Lower plot is that of two DBR lasers optically locked to two independent cavities. Sweeptime is $10 \mathrm{~s}$ for both plots.

$\sim 1.5 \mathrm{GHz}]$. Although the calculated finesse based on the mirror reflectivity should be $1000 \pm 500$, the measured ratio of fringe spacing to fringe width is only 110 , probably due to poor mode matching. Each $\phi$ mirror is also mounted on a PZT to allow optimization of the feedback phase relative to the laser. The primary output beams from the stabilized DBR lasers are combined in a beamsplitter, transmitted through an optical isolator, and then focused onto the LTG GaAs photomixer. $^{2}$ Although optically locked diode lasers are less susceptible to other, undesirable, sources of optical feedback, ${ }^{5}$ the optical isolator before the photomixer is necessary due to the high reflectivity of LTG GaAs. The THz output from the photomixer passes through a $20 \mathrm{~cm}$ gas sample cell, after which it is detected by a $4 \mathrm{~K} \mathrm{InSb}$ hotelectron bolometer. For heterodyne linewidth measurements, the LTG GaAs is replaced by a 1-GHz-bandwidth photodiode whose output is sent to a spectrum analyzer.

Figure 2 shows the effect of resonant optical feedback on the several-second timescale. The upper frame is a plot of the beatnote between free-running DBR lasers, with the spectrometer's sweep time set at $10 \mathrm{~s}$, on average, the linewidth is $90 \pm 20 \mathrm{MHz}$. The lower frame is a plot of the beatnote between two locked DBR lasers; the linewidth is 1.9 $\pm 0.7 \mathrm{MHz}$. Figure 3 shows the beatnote between two locked lasers on the 10's-of-millisecond timescale; the linewidth is $50 \pm 10 \mathrm{kHz}$. Scans that we obtained on the same timescale with free-running lasers have linewidths of $40 \pm 30 \mathrm{MHz}$. The fast linewidth is comparable to those measured by Dahmani et al. ${ }^{5}(\Delta \nu \leqslant 20 \mathrm{kHz})$ and Lee et al. ${ }^{6}(\Delta \nu=130 \mathrm{kHz})$ on diode lasers locked to separate cavities. Lee et al. also made

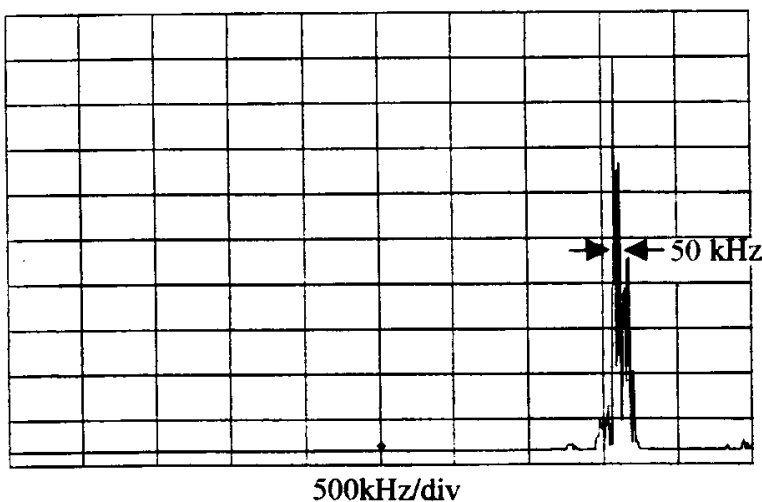

FIG. 3. The fast linewidth of two DBR lasers optically locked to two independent cavities. Sweeptime is $50 \mathrm{~ms}$. Vertical scale is linear, arbitrary.

a measurement of two lasers locked to the same cavity and obtained a linewidth of $50 \mathrm{kHz}$.

For locked DBR lasers, the cause of the slow $(\tau$ $=10 \mathrm{~s}), 2 \mathrm{MHz}$ linewidth is most likely due to temperature drifts. As for the fast $(\tau<50 \mathrm{~ms})$ linewidth of locked lasers, using a quieter power supply for the cavity PZT did improve it from about $250 \mathrm{kHz}$ to the present $50 \mathrm{kHz}$. However, the limiting factor now is probably due to variations in the feedback phase (position of $\phi$ mirror). The heterodyne output frequency is found to have an $11 \mathrm{kHz} / \mathrm{nm}$ dependence on the $\phi$-mirror position. We can roughly estimate the actual cavity finesse from this measured sensitivity of laser frequency to variations in the feedback phase by following the steadystate analysis of Laurent et al. ${ }^{7}$ (for the case of $\tau_{d}=4 \tau_{p}$; i.e., a feedback pathlength four times the cavity length),

$$
d \omega / \omega=-8 / F^{2} d \tau_{d} / \tau_{d},
$$

where $\omega$ is the laser output frequency, $F$ is the cavity finesse, $\tau_{d}$ is the round-trip delay time between the diode laser and the cavity, and $\tau_{p}$ is the round-trip time in the cavity. This gives a finesse of 40 . Considering the simplicity of the model, this agrees reasonably well with the measured value of $F=110$. Thus, increasing the cavity finesse (by improving the mode matching) should narrow the fast linewidth further.

As a demonstration of the tunability and useful intensity of the source for spectroscopy, we acquired rotational spectra of acetonitrile near $313 \mathrm{GHz}$. Figure 4 displays such a spectrum. Our single-sweep width is currently $\sim 150 \mathrm{MHz}$, limited by the self-locking range of the resonant optical feedback. This can be extended up to the cavity FSR by synchronizing the cavity length, laser current, and feedback phase during a scan. The current needs to be controlled only accurately enough that the free-running laser frequency is within the self-locking range. ${ }^{5}$

The width of the absorption lines in Fig. 4 is $4 \mathrm{MHz}$, due to a combination of acetonitrile pressure (150 mTorr) broadening and source frequency drifts. This spectrum was averaged over $100 \mathrm{~s}$ ( sampling rate $=1 \mathrm{scan} / \mathrm{s}$ ); this demonstrates the long-term stability of our source, which does not yet use any electronic feedback. The noise-to-total-power ratio in the plot shown is $6 \times 10^{-5}$. Second derivative lock-in detection was employed to enhance the signal-to-noise-ratio. The equivalent noise bandwidth (ENBW) of the lock-in am- 


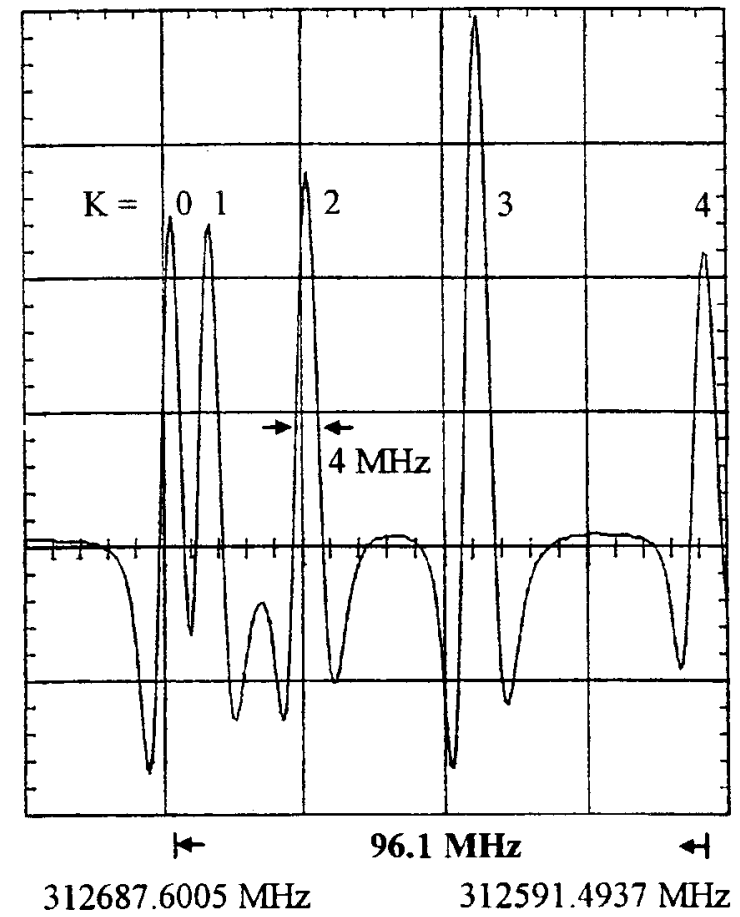

FIG. 4. Acetonitrile absorption lines. Shown above is a $K$ stack of the $J$ $=16$ transitions.

plifier was $26 \mathrm{~Hz}$. The unaveraged noise amplitude is comparable to the noise equivalent power [(NEP) $\sim 10^{-11} \mathrm{~W} \mathrm{~Hz}^{1 / 2}$, limited by the preamplifier noise] of the InSb bolometer system currently in use.

In conclusion, an all solid-state, narrow-linewidth, tunable $\mathrm{THz}$ spectrometer has been demonstrated. Dramatic future improvements in sensitivity, absolute frequency calibration, and automated scanning can be expected. For example, the photomixer's output power is currently limited by its damage threshold ( $\sim 50 \mathrm{~mW}$ of pump power); judging from the bolometer responsivity and not accounting for coupling losses, the heterodyne output is approximately $400 \mathrm{nW}$ at $300 \mathrm{GHz}$. Research is underway to raise this thermal damage threshold by cooling the device cryogenically and by using more thermally conducting substrates such as silicon. Since the output power scales quadratically with pump power, ${ }^{8}$ even a factor of three increase in the damage threshold should result in about an order of magnitude enhancement in $\mathrm{THz}$ output. In addition, resonant antennas (instead of the present broadband spiral antennas) are being designed to couple out the $\mathrm{THz}$ radiation more efficiently. On the receiving side, Si bolometers ${ }^{9}$ with NEPs in the $10^{-15} \mathrm{~W} \mathrm{~Hz}^{-1 / 2}$ range are now available to replace our present bolometer. Its lower NEP and higher responsivity should improve the ultimate sensitivity by three orders of magnitude or more.

Currently, the photomixer output frequency is calibrated using wavemeters and reference gases; a more automated and general technique is highly desired. This can be done by expanding the existing setup to a three-laser system. In one such implementation, pump laser No. 1 would be stabilized to the Cs hyperfine transition at $852 \mathrm{~nm}$; thus, the FSR of the feedback cavity would be accurately determined. Another laser, call it the transfer laser, would scan through the transmission fringes of this cavity to reach frequencies that are offset from pump laser No. 1 by integral multiples of the FSR $(n \times$ FSR1). A third laser, which would be pump laser No. 2, would be locked to a tunable cavity, and the beatnote ( $\nu_{\text {counter }}$ ) between its output and that of the transfer laser will be measured and controlled by a source-locking frequency counter. The heterodyne frequency would therefore be $(n$ $\times$ FSR $\left.1+\nu_{\text {counter }}\right)$. Since pump lasers Nos. 1 and 2 would both be actively frequency stabilized, any long-term frequency drift would be eliminated. This kind of $\mathrm{THz}$ frequency synthesis technique would also find use in nearinfrared frequency metrology by locking one of the pump lasers to known frequency standards and tuning the other one to spectral lines of interest.

The authors gratefully acknowledge L. W. Hollberg and S. B. Waltman of the National Institute of Standards and Technology at Boulder, Colorado for their generous assistance on resonant optical feedback.

${ }^{1}$ T. M. Goyette, W. Guo, F. C. De Lucia, J. C. Swartz, H. O. Everitt, B. D. Guenther, and E. R. Brown, Appl. Phys. Lett. 67, 3810 (1995).

${ }^{2}$ K. A. McIntosh, E. R. Brown, K. B. Nichols, O. B. McMahon, W. F. diNatale, and T. M. Lyszczarz, Appl. Phys. Lett. 67, 3844 (1995).

${ }^{3}$ A. S. Pine, R. D. Suenram, E. R. Brown, and K. A. McIntosh, J. Mol. Spectrosc. 175, 37 (1996)

${ }^{4}$ M. Hyodo, M. Tani, S. Matsuura, N. Onodera, and K. Sakai, Electron. Lett. 32, 1589 (1996).

${ }^{5}$ B. Dahmani, L. Hollberg, and R. Drullinger, Opt. Lett. 12, 876 (1987).

${ }^{6}$ W. David Lee, Joe C. Campbell, R. J. Brecha, and H. J. Kimble, Appl. Phys. Lett. 57, 2181 (1990).

${ }^{7} \mathrm{Ph}$. Laurent, A. Clairon, and Ch. Breant, IEEE J. Quantum Electron. 25, 1131 (1989).

${ }^{8}$ E. R. Brown, K. A. McIntosh, F. W. Smith, M. J. Manfra, and C. L. Dennis, Appl. Phys. Lett. 62, 1206 (1993).

${ }^{9}$ J. Bock, D. Chen, P. D. Mauskopf, and A. E. Lange, Space Sci. Rev. 74, 229 (1995). 